\title{
Characterization, Integration and Operation of a 100-W Solid State Amplifier in the Advanced-VIRGO Pre-Stabilized Laser System
}

\author{
Frédéric Cleva, Jean-Pierre Coulon, Fabien Kéfélian \\ ARTEMIS, Université Côte d'Azur, Observatoire de la Côte d'Azur, CNRS, F-06300 Nice, France
}

VIRGO is a 3-km-arm laser interferometer for gravitational wave detection in the $20 \mathrm{~Hz}-5 \mathrm{kHz}$ frequency band. Its second-generation version, Advanced VIRGO, aims at reducing the detector noise by a factor of ten compared to the first generation, with the progressive deployment of new technologies and equipement [1]. The first detections of gravitational waves with VIRGO were made in August 2017 [2]. For its next scientific observations with an increased sensitivity, a pre-stabilized laser system twice as powerful has been developed. It comprises a new $100-\mathrm{W}$ solid-state amplifier pumped at $878.7 \mathrm{~nm}$. It is the first time such amplifier is integrated in a gravitational wave detector, LIGO detectors using a $70 \mathrm{~W}$ amplifier pumped at $808 \mathrm{~nm}$. Power noise, long term power stability, frequency noise, beam jitter and transverse mode quality have been extensively studied and compared to Advanced VIRGO requirements.

The pre-stabilized laser system, emitting a continuous wave at $1064 \mathrm{~nm}$, is based on a $17 \mathrm{~W}$ laser oscillator, injection locked onto a low frequency noise $500 \mathrm{~mW}$ source, followed by the new $100 \mathrm{~W}$ amplifier and a $1.6-\mathrm{m}$ perimetre bow-tie shape Fabry-Perot cavity acting as a spatial and temporal mode cleaner. The optical amplifier, manufactured by neoLASE, is based on four $\mathrm{Nd}^{3+}$ : $\mathrm{YVO}_{4}$ crystals pumped longitudinally each with $50 \mathrm{~W}$ at $878,7 \mathrm{~nm}$ coming from volume Bragg grating diode lasers. The pump light is transported by 20 meters of multimode fibers deployed between two separate rooms for acoustic and electromagnetic contamination reasons. The first test and characterization campaign was performed in Nice in 2017 followed by the integration at the VIRGO site. The system has been working continuously for commissioning for more than one year.

The amplifier delivers $96 \mathrm{~W}$ with $17 \mathrm{~W}$ input and reaches $100 \mathrm{~W}$ with increased input power. No global decrease of the amplifier output power has been so far observed. The relative intensity noise spectrum (Fig. 1-a) is below $10^{-4} \times(10 \mathrm{~Hz} / \mathrm{f})^{1 / 2} \mathrm{~Hz}^{-1 / 2}$ in the detection band and limited by $13 \mathrm{~mA}$ photocurrent shot noise $\left(5 \times 10^{-9} \mathrm{~Hz}^{-}\right.$ ${ }^{1 / 2}$ ) in the 5-10 MHz band used for modulation. Further power noise reduction is realized by combining passive filtering from Fabry-Perot mode cleaners and active power stabilization with an acousto-optic modulator in transmission to reach $2 \times 10^{-9} \mathrm{~Hz}^{-1 / 2}$ in the detector band. The spectrum of the frequency noise added by the amplifier (Fig. 1-b) was measured with a heterodyne interferometer and is lower than $3 \times 10^{-1} \mathrm{~Hz} / \mathrm{Hz}^{1 / 2}$, far below the master laser oscillator. Those performances are compliant with Advanced VIRGO specifications [1].

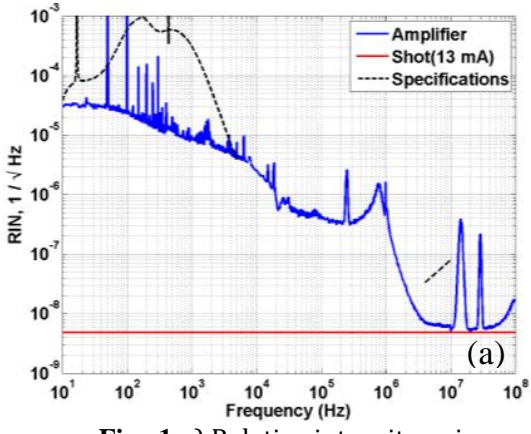

Fig. 1 a) Relative intensity noise spectrum

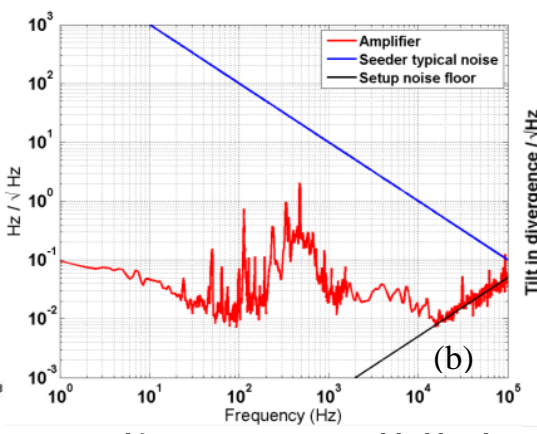

b) Frequency noise added by the amplifier ;

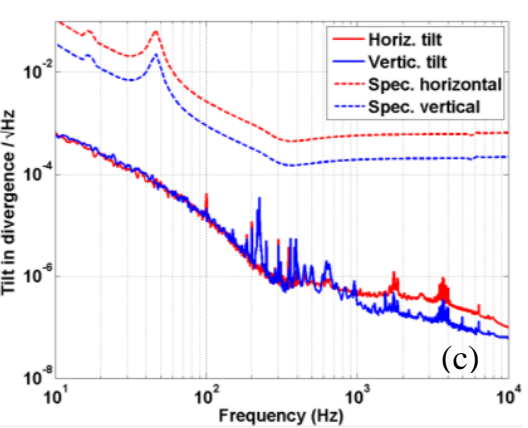

c) Beam angular jitter

The spatial beam properties of the amplifier output are another important aspect for the laser system. The output power and transverse beam profile were characterized as a function of the input beam waist size and position, and were found strongly dependent on the injection parameters. During the preliminary test and characterization campaign, the beam modal content was analysed with a Fabry-Perot cavity, giving for the optimized input beam configuration a TEM 00 content above $85 \%$. The temporal fluctuations of the beam transverse and angular (Fig. 1-c) position were measured with a pair of 4 quadrants photodiodes. The noise spectra, normalized respectively by the waist size and the divergence, are lower than $10^{-3} \times(1 \mathrm{~Hz} / \mathrm{f}) \mathrm{Hz}^{-1 / 2}$ in the detector band, one order of magnitude below the VIRGO specifications. After the complete integration on site, which required unrolling the $20-\mathrm{m}$ pump transport fibers, we noticed a reduction of the modal content. After a new optimization effort, the output power was $92 \mathrm{~W}$ with a fundamental modal content of $80 \%$. Further investigations have shown that the way the pump transport fibers are handled and stressed impacts their output beam shape and consequently may impact pump and signal overlap. Solutions to this unexpected issue are currently under investigations.

[1] F. Acernese et al., "Advanced VIRGO: a second-generation interferometric gravitational wave detector," CQG. 32, 024001 (2015). [2] P. B. Abbott et al., "GW170814 : A three-detector observation of gravitational waves from a binary black hole coalescence," Phys. Rev. Lett. 119, $141101(2017)$ 